\title{
Magnetic Characteristics of Mn-Implanted GaN Nanorods Followed by Thermal Annealing
}

\author{
Im Taek Yoon,, ${ }^{1}$ Yoon Shon, ${ }^{1}$ Younghae Kwon, ${ }^{1}$ Young S. Park, ${ }^{2}$ Chang Soo Park, ${ }^{3}$ \\ and Tae Won Kang ${ }^{1}$
}

${ }^{1}$ Quantum Functional Semiconductor Research Center, Dongguk University, Seoul 100-715, Republic of Korea
${ }^{2}$ Clarendon Laboratory, Department of Physics, University of Oxford, Parks Road, Oxford OX1 3PU, UK
${ }^{3}$ School of Electrical Engineering, Korea University, Seoul 136-701, Republic of Korea

Correspondence should be addressed to Im Taek Yoon, ityoon@dongguk.edu

Received 26 November 2011; Accepted 3 February 2012

Academic Editor: Somchai Thongtem

Copyright (C) 2012 Im Taek Yoon et al. This is an open access article distributed under the Creative Commons Attribution License, which permits unrestricted use, distribution, and reproduction in any medium, provided the original work is properly cited.

\begin{abstract}
We have investigated the magnetic and optical properties of dislocation-free vertical GaN nanorods with diameters of $150 \mathrm{~nm}$ grown on (111) Si substrates by radio-frequency plasma-assisted molecular-beam epitaxy followed by Mn ion implantation and annealing. The GaN nanorods are fully relaxed and have a very good crystal quality characterized by extremely strong and narrow photoluminescence excitonic lines near $3.47 \mathrm{eV}$. For GaMnN nanorods, it can be concluded that the ferromagnetic property of GaMnN nanorod with a Curie temperature over $300 \mathrm{~K}$ is associated with the formation of $\mathrm{Mn}_{4} \mathrm{Si}_{7}$ magnetic phase which results from the effects of magnetic and structural disorder introduced by a random incorporation and inhomogeneous distribution of $\mathrm{Mn}$ atoms in the porous layer between the nanorods that form precipitates in the Si substrate before or during the annealing step amongst the GaN nanorods.
\end{abstract}

\section{Introduction}

Dilute magnetic semiconductors (DMSs), often referred to as semimagnetic semiconductors, that are based on III$\mathrm{V}$ semiconductors have attracted a great deal of attention recently because of their potential application in spintronic devices that exploit the charge and spin of electrons [14]. The lattices of these materials consist of magnetic ions partially substituting for some of the cations, thereby inducing a local magnetic moment in the lattice and donating carriers into the system. The ferromagnetic nature of these materials is caused by the exchange interaction between localized magnetic moments introduced by the magnetic ions and the carrier spins. Therefore, for device applications, it is desirable to find materials that exhibit ferromagnetism at as high a temperature as possible. Though the concentration of $\mathrm{Mn}$ in GaMnAs has reached 10\%, the Curie temperature is no more than $150 \mathrm{~K}[5,6]$. Thus some researchers have begun to focus on a material such as one-dimensional structures (nanowires or nanorods) of nanometer scale that can provide both excellent optical and magnetic properties. One-dimensional structures (nanowires or nanorods) of nanometer-scale gallium nitride are known to have great prospects in fundamental physical science and novel technological applications $[7,8]$. Because of the large band gap and structural confinements of nanostructures, for example, the fabrication of visible and UV optoelectronic devices with relatively low power consumption is potentially feasible $[9,10]$. These studies mainly focused on zero-dimensional quantum dot and two-dimensional quantum well structures, while investigation of one-dimensional structure nanometerscale GaN could enable opportunities in understanding fundamental concepts underlying the observed electronic, optical, and mechanical properties of materials. Semiconductor quantum wires can confine excitons as in quantum dots and also exhibit other interesting phenomena, such as exciton diffusion. Therefore, GaN nanorods including Mn atoms are also expected to be used for novel spintronic device 
applications, and the structural, electronic, and magnetic properties for $\mathrm{GaN}$ nanorods and nanowires including $\mathrm{Mn}$ atoms have been investigated experimentally [11-14].

In this study, we report on the magnetic and optical properties of dilute magnetic GaN nanorods including $\mathrm{Mn}$ atoms grown on (111) substrates by radio-frequency plasmaassisted molecular beam epitaxy (rf-PAMBE) for novel device applications. High-resolution field emission scanning electron microscopy (SEM), X-ray diffraction (XRD), superconducting quantum interference device (SQUID), and photoluminescence (PL) measurement were performed to characterize the samples.

\section{Experiment}

The samples used in this study were grown on $\mathrm{Si}$ (111) substrates without any buffer layer by using rf-PAMBE. The Si substrates were degreased and etched with HF. A reconstructed $(7 \times 7)$ reflection high-energy electron diffraction pattern was obtained for the $\mathrm{Si}$ substrate after thermal treatment for $30 \mathrm{~min}$ at $1000^{\circ} \mathrm{C}$. After deoxidation, the temperature was decreased to $900^{\circ} \mathrm{C}$ and $\mathrm{GaN}$ nanorods were deposited for $3 \mathrm{~h}$. All GaN nanorods were grown at the same temperature under different III/V ratios. The rfplasma power was kept constant at $350 \mathrm{~W}$ during the growth. The Ga flux was fixed and nitrogen flow rates were varied from 1.1 to $2 \mathrm{sccm}$. All the procedures were carried out under $\mathrm{N}$-rich conditions. The maximum growth rate was $1.5 \mu \mathrm{m} / \mathrm{h}$. The formation of the GaN nanorod was confirmed by high-resolution field emission SEM. The GaN nanorods were uniformly implanted with $200 \mathrm{keV} \mathrm{Mn}^{+}$ions and dose of $5 \times 10^{16} \mathrm{~cm}^{-2}$ at a substrate temperature of $300^{\circ} \mathrm{C}$. The projected range for the depth distribution of the $\mathrm{Mn}^{+}$ions at the chosen beam energy, determined from the TRIM code, was $100 \mathrm{~nm}$, with a standard deviation of $\pm 5 \mathrm{~nm}$. After implantation, thermal annealing was performed at $800^{\circ} \mathrm{C}$ under $\mathrm{N}_{2}$ gas ambient 15,30 , and 90 minutes in a rapid thermal annealing (RTA) furnace to recover the damage. The magnetic properties were investigated using a SQUID, and a high-sensitivity $\left(10^{-8} \mathrm{emu}\right)$ alternating gradient magnetometer (AGM). In all the measurements, the magnetic field was applied perpendicular to the sample surface. XRD was used to analyze the structural and morphological properties of the as-prepared and annealed samples. The optical properties were studied by power- and temperaturedependent PL measurements. The $325 \mathrm{~nm}$ line of a HeCd laser was used as an excitation light source for the PL measurement.

\section{Results and Discussion}

Figures 1(a) and 1(b) show high-resolution scanning electron microscope images in the cross-sectional and plan view, respectively, for the GaN nanorods. The hexagonal GaN nanorods exhibit an average diameter of $150 \mathrm{~nm}$ and a density of $6.5 \times 10^{8} \mathrm{~cm}^{-2}$. These parameters are controlled by the III/V ratio during the growth process. We controlled the average diameter and density in the range of $80-190 \mathrm{~nm}$ and

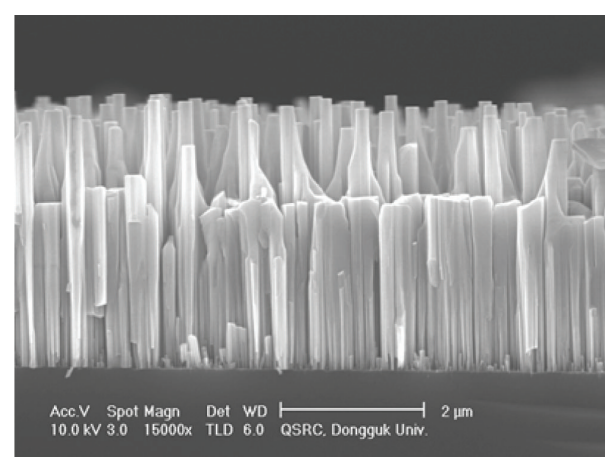

(a)

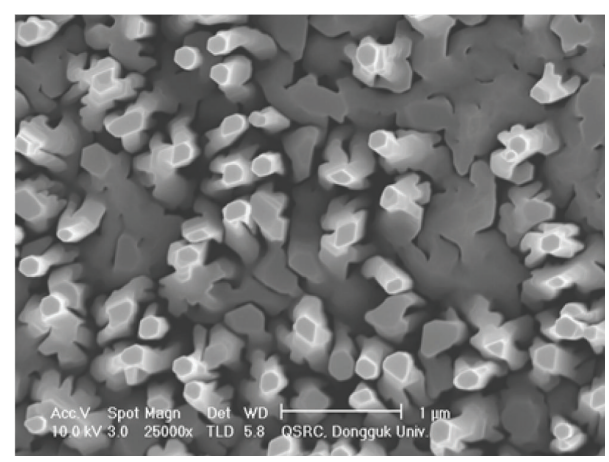

(b)

FIGURE 1: High-resolution scanning electron microscopy images of $\mathrm{GaN}$ nanorod with a diameter of $150 \mathrm{~nm}$. (a) Cross-sectional view. Scale bar is $2 \mu \mathrm{m}$. (b) Plan view. Scale bar is $1 \mu \mathrm{m}$.

$3.0-7.8 \times 10^{8} \mathrm{~cm}^{-2}$, respectively. Detailed growth conditions were reported elsewhere [15].

Figure 2 shows the XRD spectrum of the GaMnN nanorod with a diameter of $150 \mathrm{~nm}$ annealed for 15,30 , and $90 \mathrm{~min}$. The $2 \theta$ scan spectrum was obtained in the double crystal geometry. The peaks about $2 \theta=34.5,36.8$, and $72.7^{\circ} \mathrm{C}$ are attributed to the $\mathrm{GaN}(002),(101)$, and $\mathrm{GaN}(004)$ plane reflections, respectively. The diffraction peaks of GaMnN nanorod fully correspond to these of bulk GaN of a hexagonal wurtzite structure with a secondary phase. The peaks about $2 \theta=43.5$ and $81.4^{\circ} \mathrm{C}$ resulted from $\mathrm{Mn}-\mathrm{N}$ compound phase such as $\mathrm{Mn}_{4} \mathrm{Si}_{7}$ and $\mathrm{Mn}_{5} \mathrm{Si}_{3}$ precipitates. $\mathrm{Mn}_{4} \mathrm{Si}_{7}$ phase is ferromagnetic at room temperature, and $\mathrm{Mn}_{5} \mathrm{Si}_{3}$ phase is antiferromagnetic having a Néel temperature of $70-100 \mathrm{~K}[16,17]$. The $\mathrm{Mn}$ atoms might penetrate the porous layer between the nanorods and form precipitates in the Si substrate before or during the annealing step. One observes that peak intensity for the second phase decreases with increasing annealing time from 15 to $90 \mathrm{~min}$. The excitation power-dependent PL spectra of GaN nanorods with diameter of $150 \mathrm{~nm}$ at $10 \mathrm{~K}$ are shown in Figure 3(a). A strong emission line $\mathrm{A}$ around 3.47 at the high-energy side and weak emission lines $\mathrm{B}$ and $\mathrm{C}$ around $3.42,3.40 \mathrm{eV}$ are observed. The PL peak position of the main emission line $(3.47 \mathrm{eV})$ which is assigned to donor bound exciton $\left(\mathrm{D}^{0} \mathrm{X}\right.$ or $\mathrm{I}_{2}$ ) is almost unchanged during increasing of the excitation power. The full width at half maximum (FWHM) is less 


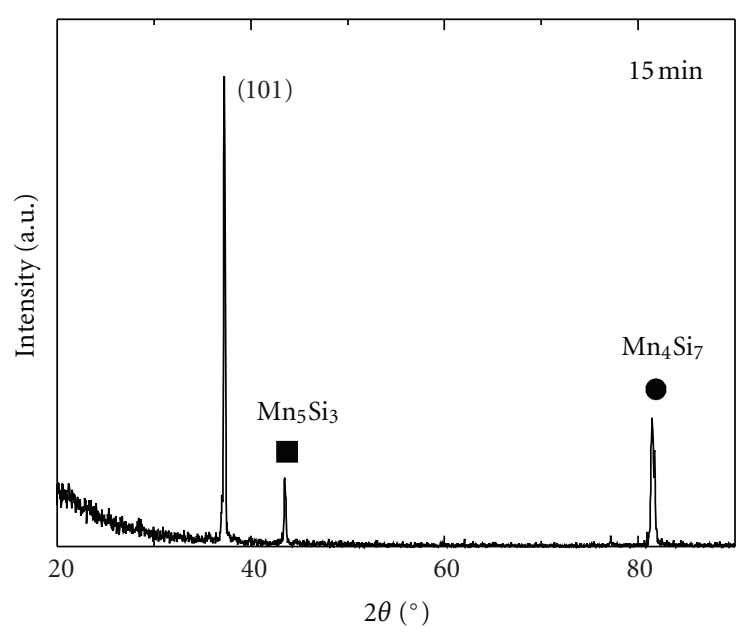

(a)

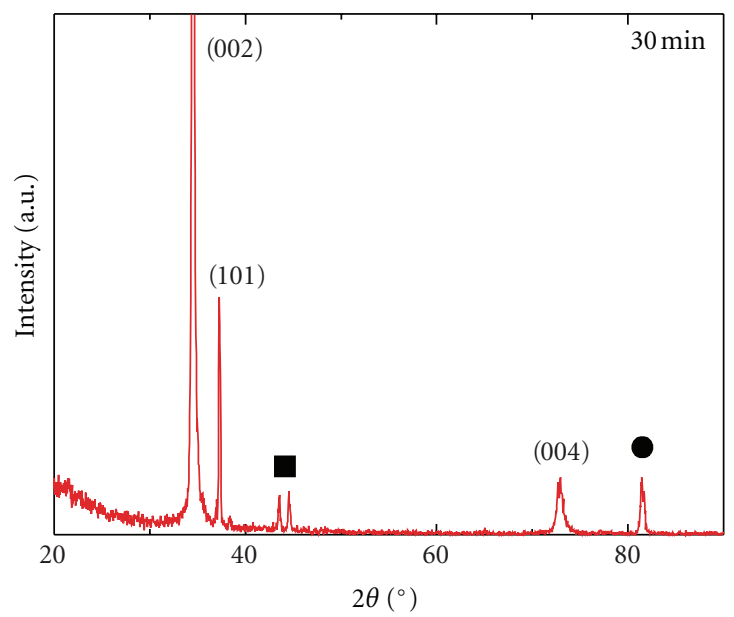

(b)

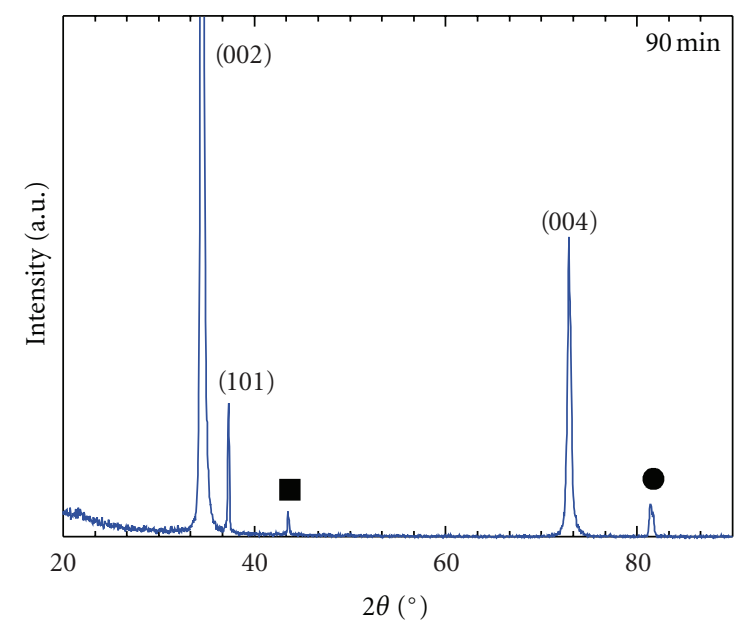

(c)

FIGURE 2: XRD pattern of GaMnN nanorod with a diameter of $150 \mathrm{~nm}$ annealed for 15, 30, and 90 min. The XRD patterns showen were characteristic of a wurtzite structure with secondary phases $\left(\mathrm{Mn}_{4} \mathrm{Si}_{7}: \mathrm{Mn}_{5} \mathrm{Si}_{3}\right.$ precipitates).

than $10 \mathrm{meV}$, which indicates high-quality GaN nanorods. The PL peak position of the emission $\mathrm{B}$ about $3.42 \mathrm{eV}$ varies from 3.41 to $3.42 \mathrm{eV}$ with an increment of excitation power. These emission lines B and C come from structural defects at the column/substrate interface, and the values agree well with the reported values by Calleja et al. [18]. Figure 3(b) shows PL spectrum of the GaMnN nanorod annealed for 15 min taken at $10 \mathrm{~K}$. The strong emission line $\mathrm{D}$ around $3.38 \mathrm{eV}$ and broad emission $\mathrm{E}$ are observed. The strong PL emission line $\mathrm{D}$ is associated with donor bound exciton corresponding to the emission line A of GaN nanorod shown in Figure 3(a). The broad emission peak E around $2.75 \mathrm{eV}$ consisted of excitonic emission and a donor-acceptor transition (DAP) related to $\mathrm{Mn}$ ion in the compact region. The emission peak $\mathrm{F}$ around $1.69 \mathrm{eV}$ is probably due to defects in the band gap of the bottom GaN compact region. Figures 4(a) and 4(b) show the magnetic field-dependent magnetization, $\mathrm{M}$, for the $\mathrm{GaMnN}$ nanorods annealed for 15,30 , and $90 \mathrm{~min}$ at $5 \mathrm{~K}$ and $300 \mathrm{~K}$, with the magnetic field applied perpendicular to the plane of the film using an AGM. Clearly elongated hysteresis loops are observed in Figure 4, indicating that a ferromagnetic structure is present. The diamagnetic background of the substrate was subtracted from the data. The sample showed coercivity of 200 Oe. M-H curves reveal that saturation magnetization of $\mathrm{GaMnN}$ nonorods decreases with increasing annealing time from 15 to $90 \mathrm{~min}$. The temperature dependence of the magnetization curve, $\mathrm{M}(\mathrm{T})$, for the GaMnN nanorods annealed for 15, 30, and $90 \mathrm{~min}$ is presented in Figure 5. A magnetic field of 3000 Oe was applied perpendicular to the film plane in the M-T measurement. It can be seen that the Curie temperature for the sample is over $300 \mathrm{~K}$. For convenience, the temperature at which the magnetic moment vanishes under a small magnetic field is taken as the Curie temperature $T_{\mathrm{C}}$, as seen in Figure 5. M-T curves reveal that magnetizations of $\mathrm{GaMnN}$ nonorods decrease with increasing annealing time from 15 to $90 \mathrm{~min}$. Annealing time-dependent behavior of $\mathrm{M}-\mathrm{H}$ curves is consistent with 


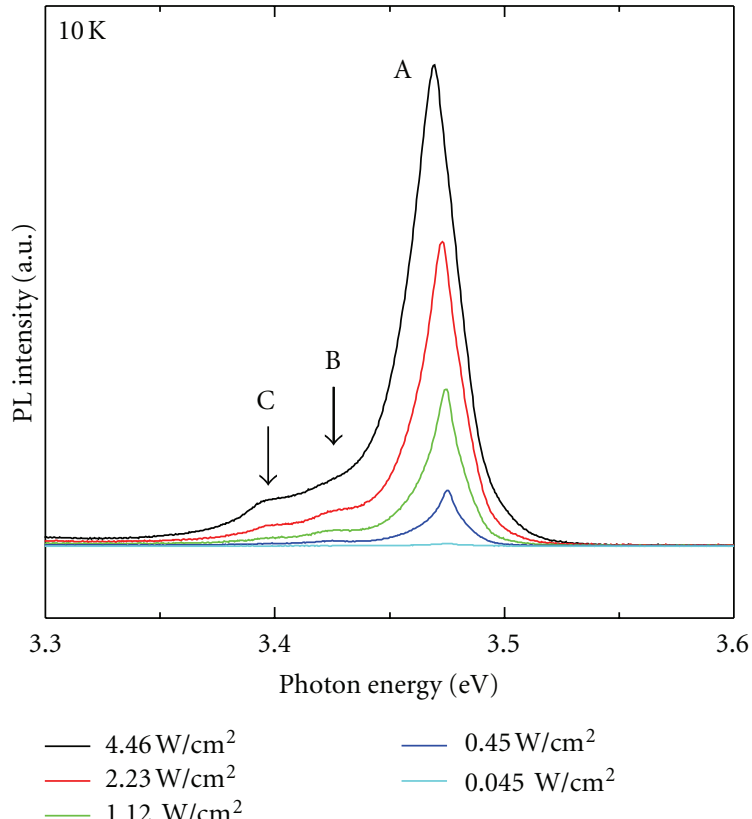

(a)

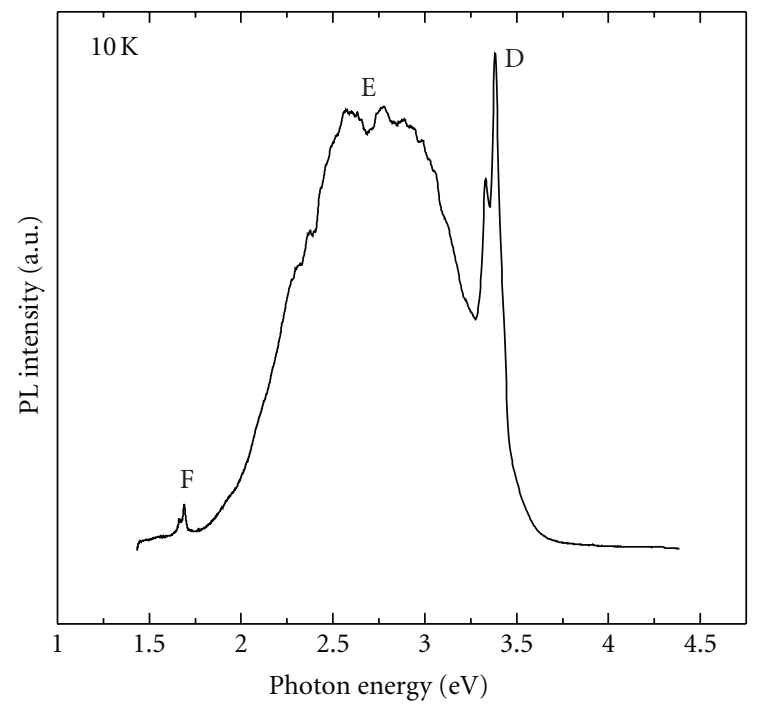

(b)

Figure 3: (a) Excitation power-dependent photoluminescence spectra of GaN nanorods with diameter of $150 \mathrm{~nm}$ at $10 \mathrm{~K}$. (b) Photoluminescence spectra of same GaMnN nanorod annealed for $15 \mathrm{~min}$ at $10 \mathrm{~K}$.

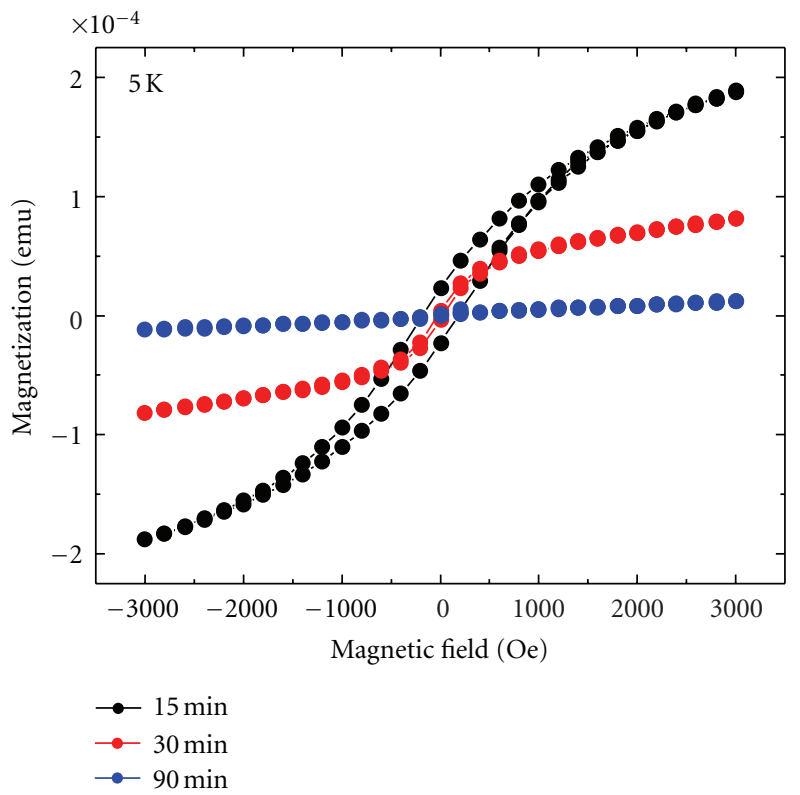

(a)

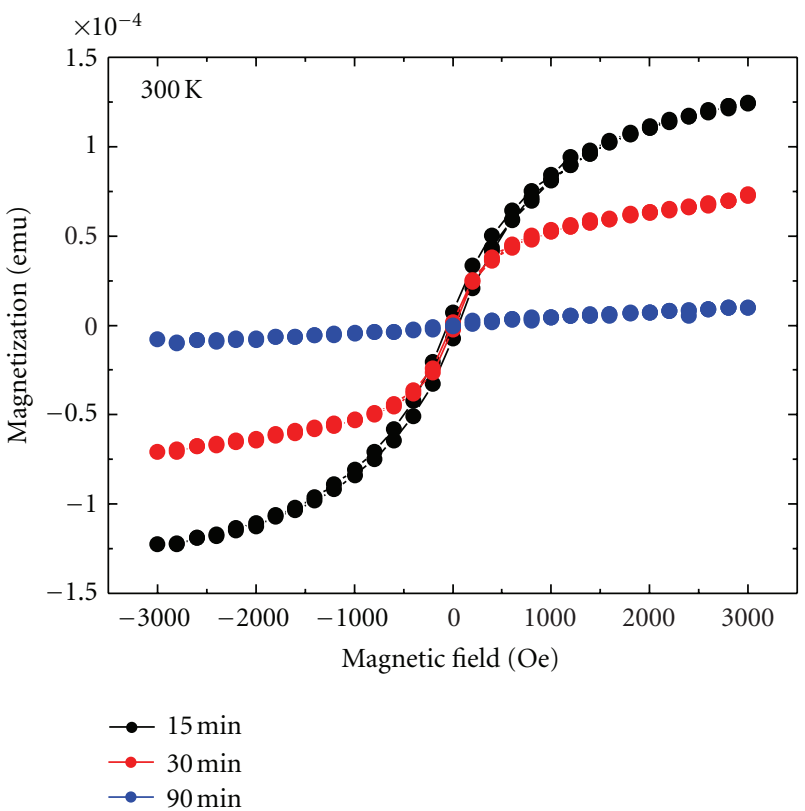

(b)

Figure 4: (a) Magnetic field-dependent magnetization M at $5 \mathrm{~K}$ for the GaMnN nanorod annealed for 15, 30, and 90 min (magnetic field perpendicular to the sample surface). (b) Magnetic field-dependent magnetization M at $300 \mathrm{~K}$ for the GaMnN nanorod annealed for 15, 30, and $90 \mathrm{~min}$. The solid line serves as a visual guide.

that of the M-T curves suggesting coexistence of multiple magnetic phases in GaMnN nanorods. In addition, annealing time-dependent behavior of $\mathrm{M}-\mathrm{H}$ and $\mathrm{M}-\mathrm{T}$ curves is also consistent with XRD data that the intensity of second phases decreases with increasing time from 15 to $90 \mathrm{~min}$. It is likely that ferromagnetic $\mathrm{Mn}_{4} \mathrm{Si}_{7}$ and anti-ferromagnetic $\mathrm{Mn}_{5} \mathrm{Si}_{3}$ phase formed during annealing process as can be seen from XRD data. A ferromagnetic $\mathrm{Mn}_{4} \mathrm{Si}_{7}$ phase is dominant with increasing annealing time from 15 to $90 \mathrm{~min}$ and anti-ferromagnetic $\mathrm{Mn}_{5} \mathrm{Si}_{3}$ phase has an effect 


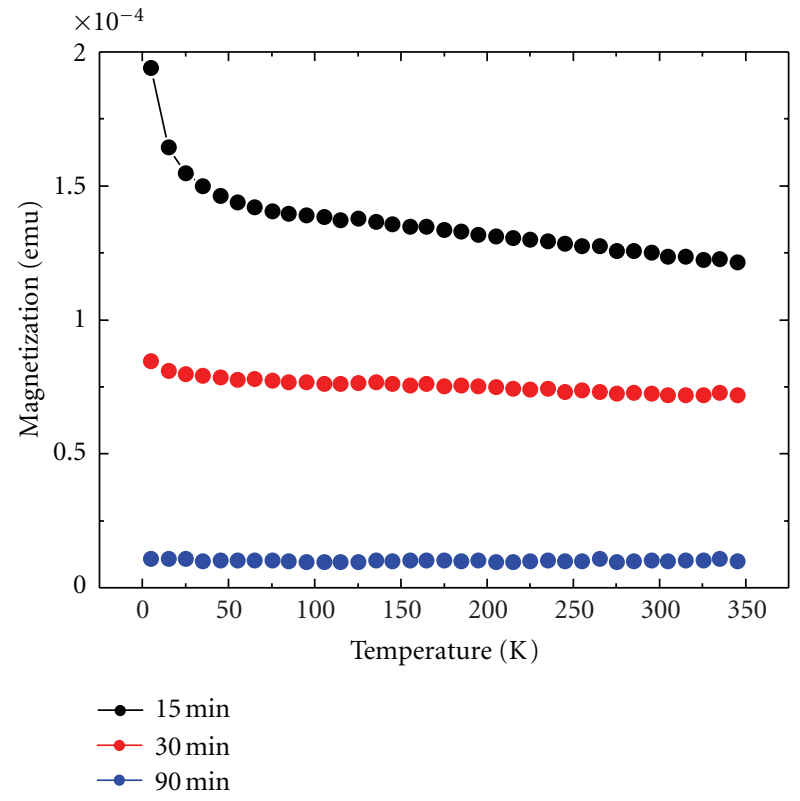

FIgURE 5: Temperature dependence of magnetization for the GaMnN nanorod annealed for 15, 30, and $90 \mathrm{~min}$ obtained by SQUID magnetometry with a magneticfield of 3000 Oe. The solid line serves as a visual guide.

decreasing the magnetization of GaMnN nanorods with increasing annealing time from 15 to $90 \mathrm{~min}$. Our data excludes the existence of other ferromagnetic compounds that have the Curie temperature of higher than $400 \mathrm{~K}$. It is well known that both GaMn and MnAs clusters present room temperature ferromagnetism [19]. Therefore, we propose that the ferromagnetism observed in the sample over $T_{\mathrm{C}}=$ $300 \mathrm{~K}$ is associated with the $\mathrm{Mn}_{4} \mathrm{~N}_{7}$ secondary phase which results from the effects of magnetic and structural disorder introduced by a random incorporation and inhomogeneous distribution of $\mathrm{Mn}$ atoms in the porous layer between the nanorods.

\section{Conclusions}

In conclusion, we have investigated the ferromagnetic and optical properties of the GaMnN nanorods with diameters of $150 \mathrm{~nm}$ by means of PL and SQUID technique. It can be concluded that the ferromagnetic property of $\mathrm{GaMnN}$ nanorods with a Curie temperature over $300 \mathrm{~K}$ is associated with the formation of $\mathrm{Mn}_{4} \mathrm{Si}_{7}$ magnetic phase which results from the effects of magnetic and structural disorder introduced by a random incorporation and inhomogeneous distribution of $\mathrm{Mn}$ atoms in the porous layer between the nanorods that form precipitates in the Si substrate before or during the annealing step amongst the GaN nanorods.

\section{Acknowledgments}

This work was supported by Basic Science Research Program through the National Research Foundation of Korea (NRF) grant funded by the Ministry of Education, Science and
Technology (MEST) (no. 2011-0025857 and 2012-0000217) as well as by Leading Foreign Research Institute Recruitment Program through NRF funded by MEST (Grant no. 201100125) and QSRC of Dongguk University.

\section{References}

[1] H. Ohno, "Making nonmagnetic semiconductors ferromagnetic," Science, vol. 281, no. 5379, pp. 951-956, 1998.

[2] H. Munekata, H. Ohno, S. Von Molnar, A. Segmuller, L. L. Chang, and L. Esaki, "Diluted magnetic III-V semiconductors," Physical Review Letters, vol. 63, no. 17, pp. 1849-1852, 1989.

[3] H. Ohno, A. Shen, F. Matsukura et al., "(Ga,Mn)As: a new diluted magnetic semiconductor based on GaAs," Applied Physics Letters, vol. 69, no. 3, pp. 363-365, 1996.

[4] H. Munekata, A. Zaslavsky, P. Fumagalli, and R. J. Gambino, "Preparation of (In,Mn)As/(Ga,Al)Sb magnetic semiconductor heterostructures and their ferromagnetic characteristics," Applied Physics Letters, vol. 63, no. 21, pp. 2929-2931, 1993.

[5] K. W. Edmonds, K. Y. Wang, R. P. Campion et al., "Hall effect and hole densities in $\mathrm{Ga}_{1-x} \mathrm{Mn}_{x} \mathrm{As}$," Applied Physics Letters, vol. 81, no. 16, p. 3010, 2002.

[6] K. W. Edmonds, K. Y. Wang, R. P. Campion et al., "High-curietemperature $\mathrm{Ga}_{1-x} \mathrm{Mn}_{x}$ As obtained by resistance-monitored annealing," Applied Physics Letters, vol. 81, no. 26, pp. 49914993, 2002.

[7] F. A. Ponce and D. P. Bour, "Nitride-based semiconductors for blue and green light-emitting devices," Nature, vol. 386, no. 6623, pp. 351-359, 1997.

[8] H. Morkoç and S. N. Mohammad, "High-luminosity blue and blue-green gallium nitride light-emitting diodes," Science, vol. 267, no. 5194, pp. 51-55, 1995.

[9] C. M. Lieber, "One-dimensional nanostructures: chemistry, physics \& applications," Solid State Communications, vol. 107, no. 11, pp. 607-616, 1998.

[10] S. Nakamura, T. Mukai, and M. Senoh, "Candela-class highbrightness InGaN/AlGaN double-heterostructure blue-lightemitting diodes," Applied Physics Letters, vol. 64, no. 13, pp. 1687-1689, 1994.

[11] X. Chen, S. J. Lee, and M. Moskovits, "Modification of the electronic properties of GaN nanowires by Mn doping," Applied Physics Letters, vol. 91, no. 8, Article ID 082109, 2007.

[12] C. S. Xue, W. J. Liu, F. Shi et al., "Fabrication of Mn-Doped GaN Nanobars," Chinese Physics Letters, vol. 27, no. 3, p. 038102, 2010.

[13] H. J. Choi, H. K. Seong, J. Chang et al., "Single-crystalline diluted magnetic semiconductor GaN:Mn nanowires," Advanced Materials, vol. 17, no. 11, pp. 1351-1356, 2005.

[14] C. Xu, J. Chun, H. J. Lee et al., "Ferromagnetic and electrical characteristics of in situ manganese-doped GaN nanowires," Journal of Physical Chemistry C, vol. 111, no. 3, pp. 1180-1185, 2007.

[15] Y. S. Park, S. H. Lee, J. E. Oh, C. M. Park, and T. W. Kang, "Self-assembled GaN nano-rods grown directly on (111) Si substrates: dependence on growth conditions," Journal of Crystal Growth, vol. 282, no. 3-4, pp. 313-319, 2005.

[16] M. Yamada, T. Goto, and T. Kanomata, "Metamagnetic properties of MnSi near the critical pressure," Journal of Alloys and Compounds, vol. 364, no. 1-2, pp. 37-47, 2004.

[17] U. Gottlieb, A. Sulpice, B. Lambert-Andron, and O. Laborde, "Magnetic properties of single crystalline $\mathrm{Mn}_{4} \mathrm{Si}_{7}$," Journal of Alloys and Compounds, vol. 361, no. 1-2, pp. 13-18, 2003. 
[18] E. Calleja, M. A. Sánchez-Garciá, F. J. Sánchez et al., "Luminescence properties and defects in $\mathrm{GaN}$ nanocolumns grown by molecular beam epitaxy," Physical Review B, vol. 62, no. 24, pp. 16826-16834, 2000.

[19] H. Katscher, "C9: Compounds of manganese with phosphorous, arsenic, antimony," in Gmelin Handbook of Inorganic and Organometallic Chemistry Vol. 56, Springer, Berlin, Germany, 8th edition, 1983. 

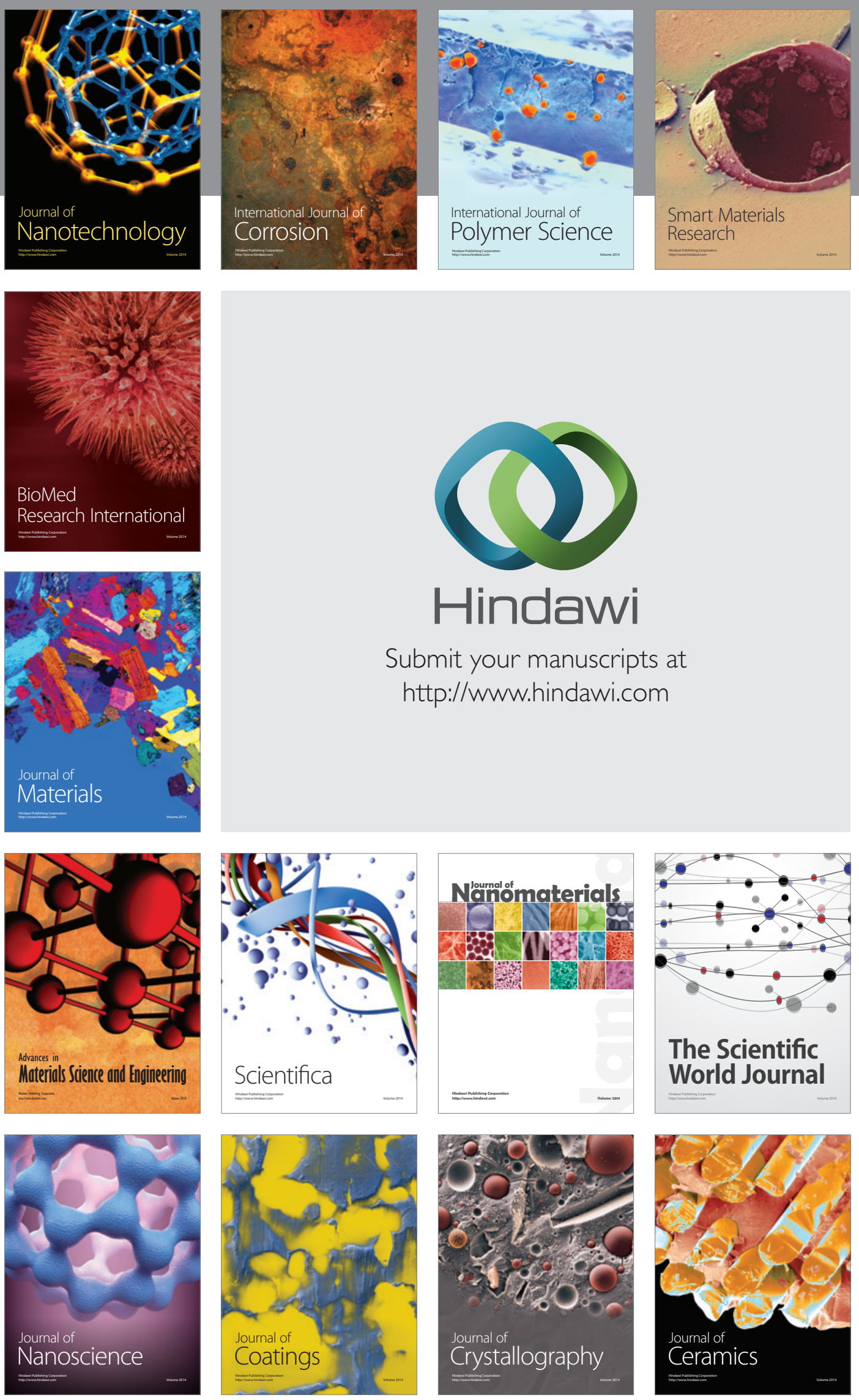

The Scientific World Journal

Submit your manuscripts at

http://www.hindawi.com

\section{World Journal}

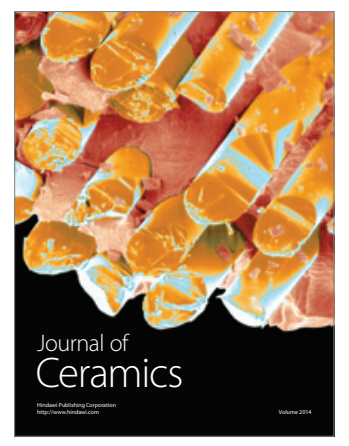

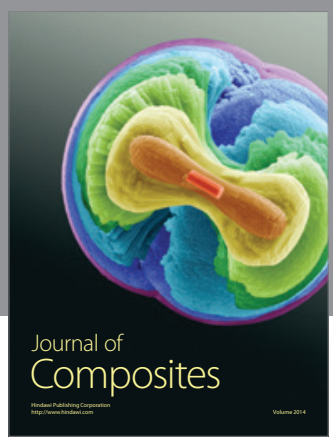
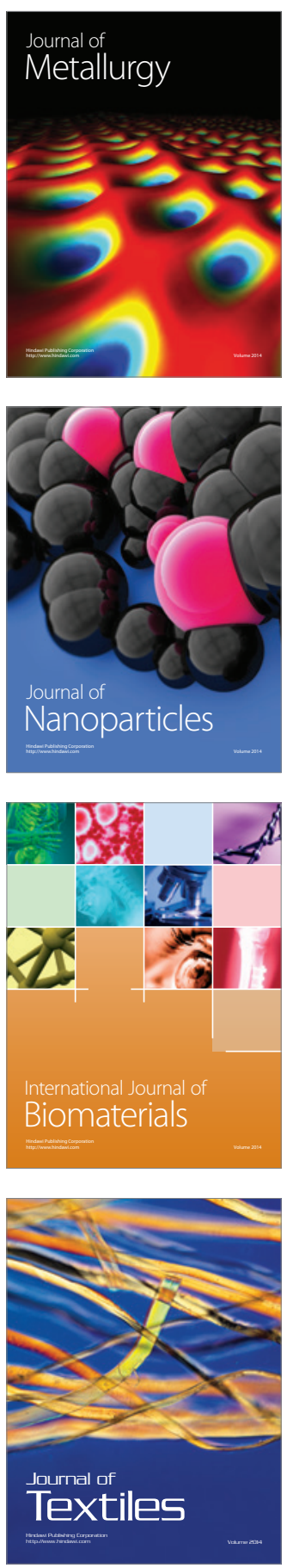